\title{
Positioning Error Analysis of Ranging-Mode Using AIS Signals in China
}

\author{
Kai Zheng, Qing Hu, and Jingbo Zhang \\ Information Science and Technology College, Dalian Maritime University, Dalian 116026, China \\ Correspondence should be addressed to Kai Zheng; kzh@dlmu.edu.cn
}

Received 24 March 2016; Revised 2 August 2016; Accepted 16 August 2016

Academic Editor: Jose R. Martinez-de Dios

Copyright ( 2016 Kai Zheng et al. This is an open access article distributed under the Creative Commons Attribution License, which permits unrestricted use, distribution, and reproduction in any medium, provided the original work is properly cited.

\begin{abstract}
In order to provide resilient position, navigation, and time (PNT) information for $E$-Navigation, the ranging-mode (R-Mode) positioning using automatic identification system (AIS) signals is encouraged. As the accuracy is the key for the positioning system, this paper investigates the position error of the R-Mode positioning based on AIS shore-based station in China. The measurement errors of Gaussian filtered minimum shift keying (GMSK) demodulation based on carrier phase locking loop are investigated in theory. The dilution of precision (DOP) for time of arrival (TOA) and time difference of arrival (TDOA) used in R-Mode positioning of AIS is discussed in two measurement mechanisms. The positioning error distributions in the North, East, and South Sea regions of China based on the existing AIS shore-based stations are evaluated. The positioning accuracy is at the meter level in the most traffic dense areas to meet the requirements for vessel navigation.
\end{abstract}

\section{Introduction}

Recently, $E$-Navigation, introduced by International Maritime Organization (IMO) and International Association of Marine Aids to Navigation and Lighthouse Authorities (IALA), has received a great deal of attention as the nextgeneration marine navigation framework. Resilient position, navigation, and time (PNT) are an essential requirement for successful implementation of E-Navigation [1]. Nowadays the global navigation satellite system (GNSS) is the main source for PNT. However, GNSS is vulnerable to deliberate, accidental, or natural radio interference $[2,3]$. A complementary system, compatible with GNSS but independent of it, is investigated by IMO, IALA, and other research organizations [4]. The automatic identification system (AIS) is recommended as the candidate for modification to add ranging-mode (R-Mode) functionality, as it has widespread distribution at present [5]. According to the International Convention for Safety of Life at Sea (SOLAS) proposed by IMO, all ships over 300 GT must be equipped with at least one AIS device to avoid maritime collision [6]. Australia [7], USA [8], and European Union [9] have deployed AIS along their coastlines and on most commercial vessels. China also has set up its shore-based automatic identification network system since 2003 [10]. The established shore-based stations can be used as the reference nodes for R-Mode positioning and no new shipboard device needs to be equipped for PNT information. Therefore, it can be an optimal choice to realize resilient PNT.

However, current AIS acts as a communication system essentially. It is used to transmit and receive maritime information among AIS equipped vessels and shore-based stations nearby, such as maritime mobile service identity (MMSI), positions, velocities, and other navigational messages. PNT information in the existing AIS is normally derived from an integral or external GNSS receiver. R-Mode positioning in AIS has been investigated mainly by Europe and China. In Europe, it is a portion of Accessibility for Shipping, Efficiency Advantages and Sustainability (ACCSEAS) project. It is focused on the feasibility study of R-Mode using AIS transmissions [11] and combining MF DGNSS, AIS, and eLoran transmissions [12]. Only theoretical research and simulation are carried out at present. In China, Maritime Safety Administration (MSA) and Dalian Maritime University have investigated technical difficulties of AIS Autonomous Positioning System (AAPS), which achieves R-Mode positioning 


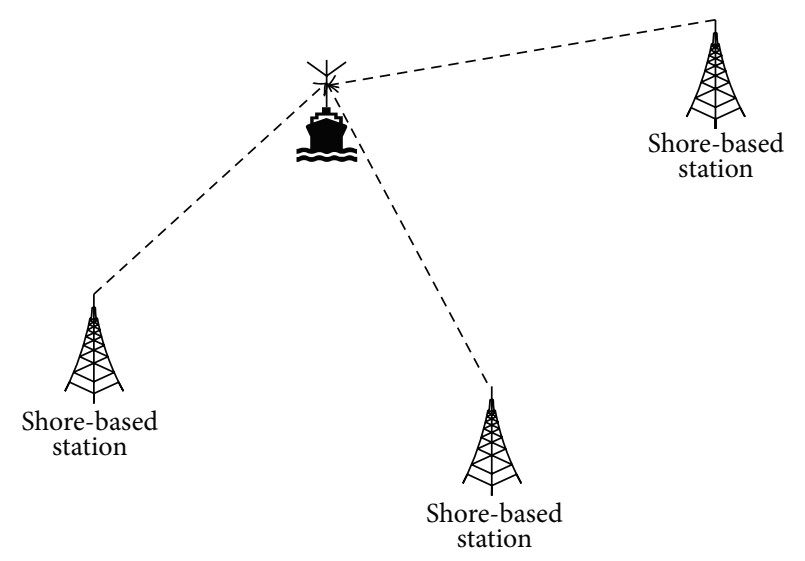

FIGURE 1: Configuration of AAPS.

using AIS signals [13-15]. On the basis of these studies in theory, the positioning testbed for the R-Mode using AIS signals has been established in Xinghai Bay of Dalian in China to verify the real-time positioning function in real sea area [16]. Based on the above research, the positioning error of RMode using AIS signals in China is analyzed in this paper, including the North China Sea, the East China Sea, and the South China Sea. It should be specially explained that the positioning error analyzed in this paper is focused on the measurement error and the dilution of precision (DOP). The measurement error discussed here is assuming transmission through an additive white Gaussian noise (AWGN) channel, without considering the additional secondary factors (ASFs), the multipath, and other interference.

The rest of the paper is organized as follows. Section 2 investigates the principle of R-Mode positioning using AIS signals. In Section 3, Gaussian filtered minimum shift keying (GMSK) demodulation based on carrier phase locking loop is presented and the measurement errors are theoretically discussed. In Section 4, DOP for time of arrival (TOA) and time difference of arrival (TDOA) in R-Mode positioning of AIS are discussed in different conditions. Section 5 analyzes positioning accuracy based on the existing AIS shore-based stations in China sea area. Finally, conclusions are made in Section 6.

\section{Principle of R-Mode Positioning in AIS}

AIS acts as a broadcast system providing identification of targets together with the static and dynamic information. AIS enhanced with ranging-mode position, called AAPS, can provide the range information from the shore-based station to the vessel used for estimating the position of the coastal vessel. As illustrated in Figure 1, AAPS usually consists of many AIS shore-based stations and a shipborne AIS device. For all the AIS shore-based stations, the time synchronization is required. Meanwhile, shipborne AIS device need not be synchronous with shore-based stations.

In AAPS, the precision position of shore-based stations as the reference nodes should be known by the vessel for its position estimation. The primary messages available from

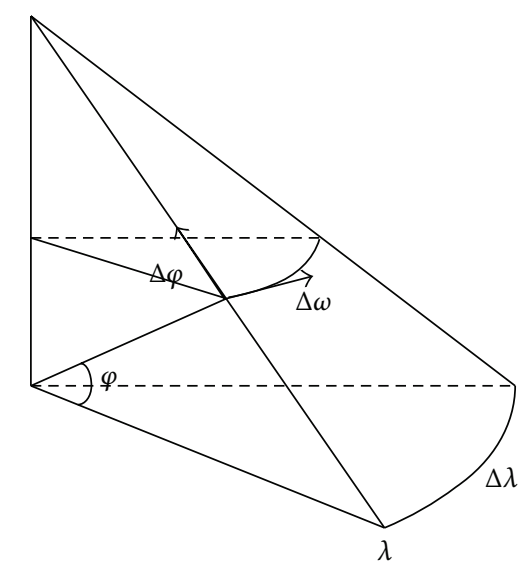

FIGURE 2: Relationship between $(\varphi, \lambda)$ and $(\Delta \varphi, \Delta \omega)$.

the shore-based station are message 4 in AIS at present, which is the Universal Time Coordinated (UTC) time and position report. Although the shore-based station normally transmits message 4 with a reporting interval of $10 \mathrm{~s}$, the shipborne AIS device could store the position information of the shore-based station once message 4 has been received and use it for the subsequent processing. Shipborne AIS device can receive very high frequency (VHF) AIS signal from different shore-based stations. The range information is obtained by measuring the signal propagation delay in the R-Mode positioning based on AIS. Both time of arrival (TOA) [17] and time difference of arrival (TDOA) [18] can be utilized. The location of the vessel can be estimated using the latitude and longitude coordinates of the shore-based stations by TOA and TDOA $[19,20]$. As shown in Figure 2, the latitude and longitude of the vessel are denoted by $(\varphi, \lambda)$. For convenience, the changes in latitude and longitude are denoted by the horizontal and vertical increments $(\Delta \varphi, \Delta \omega)$.

The coordinate of the vessel positon and increments satisfies the following equations:

$$
\begin{aligned}
& \varphi^{\prime}=\varphi+\Delta \varphi \\
& \lambda^{\prime}=\lambda+\Delta \omega \sec \varphi .
\end{aligned}
$$

The positioning equation is written in matrix form as follows:

$$
\operatorname{AdX}=\mathbf{L}
$$

where $\mathbf{A}$ and $\mathbf{L}$ are called location matrix and observation matrix, respectively:

$$
\mathbf{d X}=\left[\begin{array}{ll}
d \varphi & d \omega
\end{array}\right]^{\mathrm{T}} .
$$

The least square solution is usually used to estimate the corrections $(\Delta \varphi, \Delta \omega)$, which can be written as

$$
\mathbf{d X}=\left(\mathbf{A}^{\mathrm{T}} \mathbf{A}\right)^{-1} \mathbf{A}^{\mathrm{T}} \mathbf{L} .
$$

Assuming the measurement error is very small, the effects on the linearization of the positioning equation can be neglected. 
If the measurement error term can be retained, positioning equation (2) can be rewritten as

$$
\mathbf{A}\left[\mathbf{d X}+\varepsilon_{\mathbf{X}}\right]=\mathbf{L}+\boldsymbol{\varepsilon},
$$

where $\boldsymbol{\varepsilon}_{\mathbf{x}}$ is the positioning error vector and $\boldsymbol{\varepsilon}$ denotes the measurement error vector. The covariance of the positioning error is

$$
\operatorname{cov}\left(\boldsymbol{\varepsilon}_{\mathbf{x}}\right)=E\left(\boldsymbol{\varepsilon}_{\mathbf{x}} \boldsymbol{\varepsilon}_{\mathbf{x}}^{\mathrm{T}}\right)=\left(\mathbf{A}^{\mathrm{T}} \mathbf{A}\right)^{-1} \sigma^{2},
$$

where $\sigma$ is the common standard deviation of the measurement error; a coefficient matrix $\left(\mathbf{A}^{\mathrm{T}} \mathbf{A}\right)^{-1}$ is symmetric, whose element contains the geometric accuracy and the correlated information of all the solutions.

As the existing AIS is a communication system, there are technical difficulties for the R-Mode positioning using AIS signals. The first technical difficulty is the measurement of the range using AIS signals. Generally speaking, signals are continuous and periodic provided by the traditional positioning system for the range measurement, such as the pulse-envelope measurement for E-Loran [21] and carrier phase measurement for GNSS [22]. There are two dedicated VHF frequencies used for AIS, that is, channel $87 \mathrm{~B}$ $(161.975 \mathrm{MHz})$ and channel 88B (162.025 MHz). AIS signals use a time-division multiple access (TDMA) scheme to share the VHF frequency, known as the VHF Data Link (VDL). The VDL is arranged into frames, which are repeated every one minute. And one frame is divided into 2250 time slots. Each AIS station transmits a message in one of the time slots; at the same time it listens to all the time slots and reads the received message information. Therefore, AIS signals from shore-based stations do not have the continuous time slots and periodicity. Therefore, it is difficult to obtain range information by tracking the noncontinuous AIS signals. GMSK demodulation based on carrier phase locking loop given in Section 3 can be utilized to solve this problem. Secondly, the existing shore-based stations are not established for positioning specifically. Thus, the DOP of the shore-based stations is discussed in Section 4. Furthermore, the next generation of AIS, called the VHF Data Exchange System (VDES), has been developing rapidly and popularized by IMO [23]. There are more channels and frequencies that can be used. In addition, more shore-based stations will be constructed all over the world. Thus the performance of R-Mode positioning based on the AIS could be improved, including the positioning accuracy and the update frequency.

\section{Measurement Error Analysis}

As AIS is a TDMA system, the signal is noncontinuous and nonperiodic. In order to obtain the range from the shorebased station to the vessel by measuring the signal transmission delay, we use GMSK demodulation based on carrier phase locking loop (PLL) to track AIS signals. The time of bit transition and the carrier phases are both estimated. The GMSK modulation and demodulation system has been built based on Simulink and FPGA to verify the function of the range measurement. Then the performance of measurement is investigated in the view of theory.
3.1. GMSK Demodulation. The noncoherent demodulation is widely used in traditional shipborne device for communication. The requirement for the decision time of each bit transition is not strict. It cannot satisfy the accuracy requirement for the positioning system, because the maximum decision time measurement error of one-bit transition may be one period theoretically. Thus, the frequency and the phase of AIS carrier signals should be both tracked for AAPS so that both the time of bit transitions and the carrier phases should be estimated in realization.

The period of the time slot is $26.67 \mathrm{~ms}$. The corresponding distance is $8001 \mathrm{~km}$. It far exceeds the transmission distance of AIS signals. Therefore, the transmission time from the shorebased station should be within one time slot. The number of the time slot can be unpacked according to the link layer of AIS signals [24]. At the same time, according to the slot phase synchronization, the frame synchronization, and the training sequence, the frequency and the phase of the carrier are tracked and the exact transmission time in a time slot is measured. The method is called GMSK demodulation based on carrier phase tracking [25]. The block diagram of GMSK demodulation based on carrier phase locking loop is shown in Figure 3.

The intermediate frequency (IF) digital signal in the shipborne AIS device is written as

$$
S(t)=A \sin (2 \pi f t+\varphi)
$$

where $A$ denotes the signal amplitude; $f$ is the carrier frequency of the input digital IF, which is varying with time; $\varphi$ denotes the initial carrier phase. After $90^{\circ}$ phase shifts, the input IF signal produces in-phase $(I)$ and quadraphase (Q) form. By mixing with the local carrier, the $I$ and $Q$ signals in (8) are generated with both the upper and the lower sidebands:

$$
\begin{aligned}
& I(t)=\frac{A}{2} \cdot\{\sin [2 \pi(\widehat{f}+f) t+(\widehat{\varphi}+\varphi)] \\
& +\sin [2 \pi(\widehat{f}-f) t+(\widehat{\varphi}-\varphi)]\} \\
& Q(t)=\frac{A}{2} \cdot\{\sin [2 \pi(\widehat{f}+f) t+(\widehat{\varphi}+\varphi)] \\
& -\sin [2 \pi(\widehat{f}-f) t+(\widehat{\varphi}-\varphi)]\},
\end{aligned}
$$

where $\widehat{f}$ and $\widehat{\varphi}$ are the frequency and the phase of the carrier, respectively. $I$ subtracted from $Q$ can remove the upper sideband and select the lower sideband; that is,

$$
S_{I Q}(t)=A \sin [2 \pi \Delta f t+\Delta \varphi],
$$

where $\Delta f$ is a frequency offset, due to mismatch of the local oscillator in the demodulation process; $\Delta \varphi$ is an unknown phase, due to the phase offset between the local oscillator at the receiver and the signal carrier. As PLL, $S_{I Q}(t)$ is processed by the discriminator and the filter of the tracking loop operated, then the baseband signal can be obtained.

Each zero crossing location of the baseband signal corresponds to the location of the bit transition in AIS signals. Each zero crossing of the baseband signal can be detected 


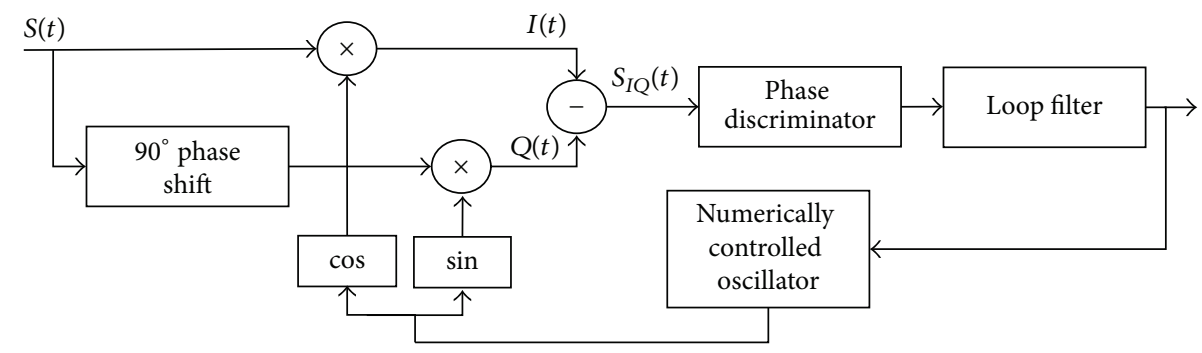

FIGURE 3: Block diagram of GMSK demodulation based on carrier phase locking loop.

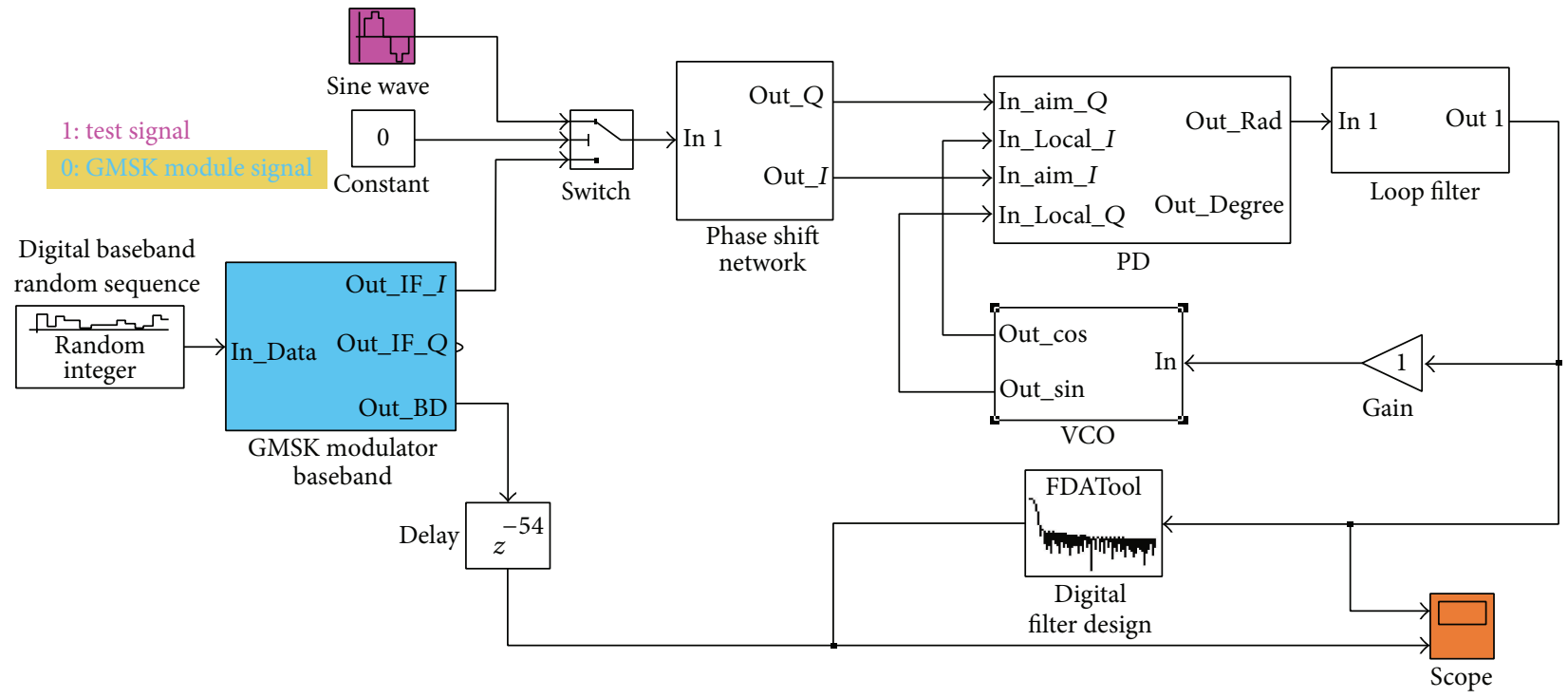

FIGURE 4: Structure diagram of GMSK modulation and demodulation verification system.

in GMSK demodulation based on carrier phase tracking. Therefore, exact transition time from one bit to the next bit can be measured in GMSK demodulation. The transmission time in the time slot can be calculated.

3.2. Simulation Verification. In order to verify the above demodulation scheme and optimize the loop parameters, the GMSK modulation and demodulation verification system is built on the Simulink platform. The simulation diagram is shown in Figure 4.

The demodulation results are illustrated in Figure 5. The top curve represents the output from the Gauss filter before modulation. The middle curve shows the GMSK modulation signal. The bottom curve is the output after GMSK demodulation based on carrier PLL. It can be seen that the GMSK signal is effectively demodulated.

The special counter is utilized to record the bit transition time of serial data signals. It starts from zero at the beginning of a slot. The exact transition time in one time slot can be measured in GMSK demodulation based on FPGA circuit.

3.3. Performance Analysis. A Cramér-Rao Bound (CRB) on the performance of any estimator of time delay from the bit edges through an AWGN channel is developed in [26]. When

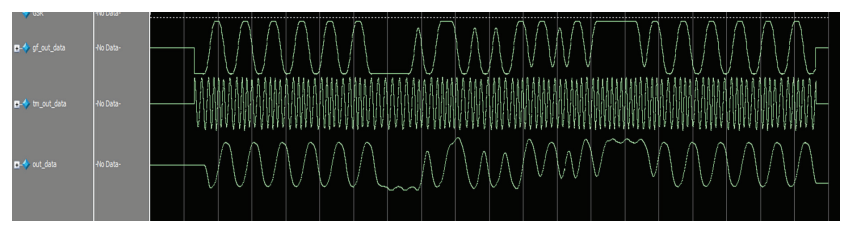

FIGURE 5: Demodulation result based on FPGA.

the transmitted data is assumed to be random, a lower bound to the CRB for the time delay can be simplified to

$$
\operatorname{CRB}(\tau)=\frac{N_{0}}{E\left\{\int_{0}^{T_{0}}|\partial s(t, \tau, \Delta f, \Delta \varphi) / \partial \tau|^{2} d t\right\}},
$$

where the spectral density of AWGN is $N_{0} / 2 ; T_{0}$ is the entire observation interval; $\tau$ is the time delay, which is the term of interest for ranging; and $E\{\cdot\}$ is the statistical expectation operator. For the GMSK, this bound can be simplified to [11]

$$
\mathrm{CRB}_{G}(\tau)=\frac{1.3982 \times 10^{-20}}{L P_{r}},
$$


where $P_{r}$ is the received power in $\mathrm{mV} ; L$ is the number of bits.

$$
L=\frac{T_{0}}{T_{S}}
$$

For AIS, $T_{S}$ equals $1 / 9600$.

For a point-to-point link, it is preferable to calculate the free-space attenuation loss as follows [27]:

$$
L_{b f}=10 \lg \left(\frac{P_{t}}{P_{r}}\right)=32.4+20 \lg D+20 \lg F,
$$

where $P_{t}$ is the transmitted power in the unit of $\mathrm{mV} ; D$ is the transmission distance in the unit of $\mathrm{km} ; F$ is the frequency in the unit of MHz. Transmitted power $P_{t}$ of AIS shorebased station usually is $25 \mathrm{~W}$. The nominal carrier center frequency $f$ is $162 \mathrm{MHz}$ [28]. Thus, the relationship between the received power and the transmission distance is

$$
P_{r}=\frac{9 P_{t}}{(40 \pi D F)^{2}}=\frac{5.4292 \times 10^{-4}}{D^{2}} .
$$

Thus, standard deviation of measurement in time can be written as follows:

$$
\sigma_{\mathrm{GMSK}} \geq \sqrt{\mathrm{CRB}_{G}(\tau)}=\frac{5.0750 \times 10^{-9} \mathrm{D}}{\sqrt{L}} .
$$

Measurement error is increasing with transmission distances. Meanwhile, the longer the observation measured data $L$ is, the smaller the measurement error is.

\section{Dilution of Precision Analysis}

The shore-based station of AAPS is considered as the reference node. Positioning error, in terms of DOP, is related to the distribution of the reference nodes. As the shore-based stations are not established for positioning specifically, DOP for TOA and TDOA used in AAPS is discussed in this section. The clock synchronization is difficult to achieve between the AIS shore-based station and the shipborne AIS device. Therefore, DOP of R-Mode positioning in AIS is discussed in this paper without time synchronization. We compare DOP of TOA and TDOA by comparing their trace of the coefficient matrix according to the positioning equation.

4.1. DOP of TDOA. According to (6), the covariance matrix for TDOA is given by

$$
\operatorname{cov}\left(\boldsymbol{\varepsilon}_{\mathbf{x T D O A}}\right)=\left(\mathbf{G}^{\mathrm{T}} \mathbf{G}\right)^{-1} \sigma_{\Delta \rho}^{2},
$$

where $\sigma_{\Delta \rho}$ is the standard deviation of the measurement error. The location matrix for TDOA is

$$
\mathbf{G}=\left[\begin{array}{cc}
-\sin \theta_{m}+\sin \theta_{1} & -\cos \theta_{m}+\cos \theta_{1} \\
\vdots & \vdots \\
-\sin \theta_{m}+\sin \theta_{n-1} & -\cos \theta_{m}+\cos \theta_{n-1}
\end{array}\right] .
$$

Meanwhile, under the ideal condition TOA with the time synchronization, the location matrix $\mathbf{h}$ is

$$
\mathbf{h}=\left[\begin{array}{cc}
-\sin \theta_{1} & -\cos \theta_{1} \\
\vdots & \vdots \\
-\sin \theta_{n} & -\cos \theta_{n}
\end{array}\right]
$$

Thus

$$
\mathbf{G}=\mathbf{S h},
$$

where $\mathbf{S}$ is called a transformation matrix. It is defined as follows:

$$
\mathbf{S}=\left[\begin{array}{ll}
\mathbf{e}_{n-1} & -\mathbf{I}_{n-1}
\end{array}\right],
$$

where $\mathbf{e}_{n-1}=\left[\begin{array}{lll}1 & \cdots & 1\end{array}\right]_{(n-1) \times 1}^{\mathrm{T}} . \mathbf{I}_{n-1}$ is the identity matrix of order $(n-1)$. Covariance matrix (16) can be rewritten as

$$
\begin{aligned}
\operatorname{cov}\left(\boldsymbol{\varepsilon}_{\mathbf{x T D O A}}\right) & =\left(\mathbf{h}^{\mathrm{T}} \mathbf{S}^{\mathrm{T}} \mathbf{S h}\right)^{-1} \sigma_{\Delta \rho}^{2} \\
& =\left(\mathbf{h}^{\mathrm{T}} \mathbf{I}_{n} \mathbf{h}+\mathbf{h}^{\mathrm{T}} \mathbf{P h}\right)^{-1} \sigma_{\Delta \rho}^{2},
\end{aligned}
$$

where

$$
\mathbf{P}=\left[\begin{array}{cc}
n-1 & -\mathbf{e}_{n-1}^{\mathrm{T}} \\
-\mathbf{e}_{n-1} & \mathbf{0}_{n-1}
\end{array}\right]
$$

4.2. DOP of TOA. The covariance matrix for TOA without the time synchronization is given by

$$
\operatorname{cov}\left(\boldsymbol{\varepsilon}_{\mathbf{x} t \mathrm{TOA}}\right)=\left(\mathbf{H}^{\mathrm{T}} \mathbf{H}\right)^{-1} \sigma_{\rho}^{2},
$$

where $\sigma_{\rho}$ is the standard deviation of the measurement error. The location matrix $\mathbf{H}$ is

$$
\mathbf{H}=\left[\begin{array}{ll}
\mathbf{h} & \mathbf{e}_{n}
\end{array}\right] .
$$

Thus

$$
\mathbf{H}^{\mathrm{T}} \mathbf{H}=\left[\begin{array}{cc}
\mathbf{h}^{\mathrm{T}} \mathbf{h} & \mathbf{h}^{\mathrm{T}} \mathbf{e}_{n} \\
\mathbf{e}_{n}^{\mathrm{T}} \mathbf{h} & \mathbf{e}_{n}^{\mathrm{T}} \mathbf{e}_{n}
\end{array}\right]=\left[\begin{array}{cc}
\mathbf{h}^{\mathrm{T}} \mathbf{h} & \mathbf{h}^{\mathrm{T}} \mathbf{e}_{n} \\
\mathbf{e}_{n}^{\mathrm{T}} \mathbf{h} & n
\end{array}\right] .
$$

According to the inverse of the block matrix, we know that

$$
\left(\mathbf{H}^{\mathrm{T}} \mathbf{H}\right)^{-1}=\left[\begin{array}{ll}
\mathbf{H}_{11} & \mathbf{H}_{12} \\
\mathbf{H}_{21} & \mathbf{H}_{22}
\end{array}\right]^{-1} .
$$

The covariance matrix of the positioning error for TOA is

$$
\begin{aligned}
\operatorname{cov}\left(\boldsymbol{\varepsilon}_{\mathbf{x T O A}}\right) & =\left(\mathbf{H}_{11}-\mathbf{H}_{12} \mathbf{H}_{22}^{-1} \mathbf{H}_{21}\right)^{-1} \sigma_{\rho}^{2} \\
& =n\left(\mathbf{G}^{\mathrm{T}} \mathbf{J G}\right)^{-1} \sigma_{\rho}^{2},
\end{aligned}
$$

where

$$
\mathbf{J}=\left[\begin{array}{cccc}
n-1 & -1 & \cdots & -1 \\
-1 & n-1 & & -1 \\
\vdots & \vdots & \ddots & \vdots \\
-1 & -1 & \cdots & n-1
\end{array}\right]_{(n-1) \times(n-1)}
$$

Therefore, the positioning error for TOA without the time synchronization is only determined by the matrix $\left(\mathbf{G}^{\mathrm{T}} \mathbf{J G}\right)^{-1}$. 
4.3. Comparison of TDOA and TOA without the Time Synchronization. Two situations are discussed in the following according to measurement parameters.

Situation 1. The variances of the measurement errors are equal to the assumption on the same measurement of TOA and TDOA:

$$
\sigma_{\rho}^{2}=\sigma_{\Delta \rho}^{2}=\sigma^{2}
$$

Hence,

$$
\begin{aligned}
& \operatorname{cov}\left(\boldsymbol{\varepsilon}_{\mathbf{x} T O A}\right)-\operatorname{cov}\left(\boldsymbol{\varepsilon}_{\mathbf{x} T D O A}\right) \\
& =\left[n\left(\mathbf{G}^{\mathrm{T}} \mathbf{J G}\right)^{-1}-\left(\mathbf{G}^{\mathrm{T}} \mathbf{G}\right)^{-1}\right] \sigma^{2} .
\end{aligned}
$$

From (28) and (20), we can get

$$
\mathbf{J}=n\left(\mathbf{S S}^{\mathrm{T}}\right)^{-1}
$$

thus,

$$
\begin{aligned}
\operatorname{cov} & \left(\boldsymbol{\varepsilon}_{\mathbf{x} T O A}\right)-\operatorname{cov}\left(\boldsymbol{\varepsilon}_{\mathbf{x T D O A}}\right) \\
= & {\left[\left(\mathbf{G}^{\mathrm{T}}\left(\mathbf{S S}^{\mathrm{T}}\right)^{-1} \mathbf{G}\right)^{-1}-\left(\mathbf{G}^{\mathrm{T}} \mathbf{G}\right)^{-1}\right] \sigma^{2} } \\
= & \frac{1}{\left|\mathbf{G}^{\mathrm{T}} \mathbf{G}\right|}\left[\begin{array}{ll}
c_{11} & c_{12} \\
c_{21} & c_{22}
\end{array}\right] \sigma^{2} .
\end{aligned}
$$

In the R-Mode positioning for AIS, the vessel could receive signals from three shore-based stations. In this situation,

$$
\begin{aligned}
c_{11} & =\sin ^{2} \gamma_{2}+\sin ^{2} \gamma_{3}-2 \sin \gamma_{2} \sin \gamma_{3} \\
& =\left(\sin \gamma_{2}-\sin \gamma_{3}\right)^{2} \geq 0 \\
c_{22} & =\cos ^{2} \gamma_{2}+\cos ^{2} \gamma_{3}-2 \cos \gamma_{2} \cos \gamma_{3} \\
& =\left(\cos \gamma_{2}-\cos \gamma_{3}\right)^{2} \geq 0 .
\end{aligned}
$$

Thus, the positioning error for TOA is always greater than TDOA under the condition with the same AIS shore-based stations. The performance using TDOA method is always better than TOA.

Situation 2. The range difference used in TDOA method is obtained according to the measured range from different AIS shore-based stations. Assuming variances of the range measurement errors are all equal to $\sigma^{2}$, then the variances of the measurement errors of TDOA and TOA satisfy

$$
\sigma_{\Delta \rho}^{2}=\mathbf{S} \sigma_{\rho}^{2} \mathbf{S}^{\mathrm{T}}=\mathbf{S} \sigma^{2} \mathbf{S}^{\mathrm{T}}
$$

When the number of the shore-based stations is three,

$$
\begin{aligned}
& \operatorname{cov}\left(\boldsymbol{\varepsilon}_{\mathbf{x T O A}}^{\prime}\right)-\operatorname{cov}\left(\boldsymbol{\varepsilon}_{\mathbf{x} T D O A}\right) \\
& =\left(\mathbf{G}^{\mathrm{T}}\left(\mathbf{S S}^{\mathrm{T}}\right)^{-1} \mathbf{G}\right)^{-1} \sigma^{2}-\left(\mathbf{G}^{\mathrm{T}} \mathbf{G}\right)^{-1}\left(\mathbf{S S}^{\mathrm{T}}\right) \sigma^{2}=0 .
\end{aligned}
$$

Thus, the performance of TDOA is the same as TOA in this situation.

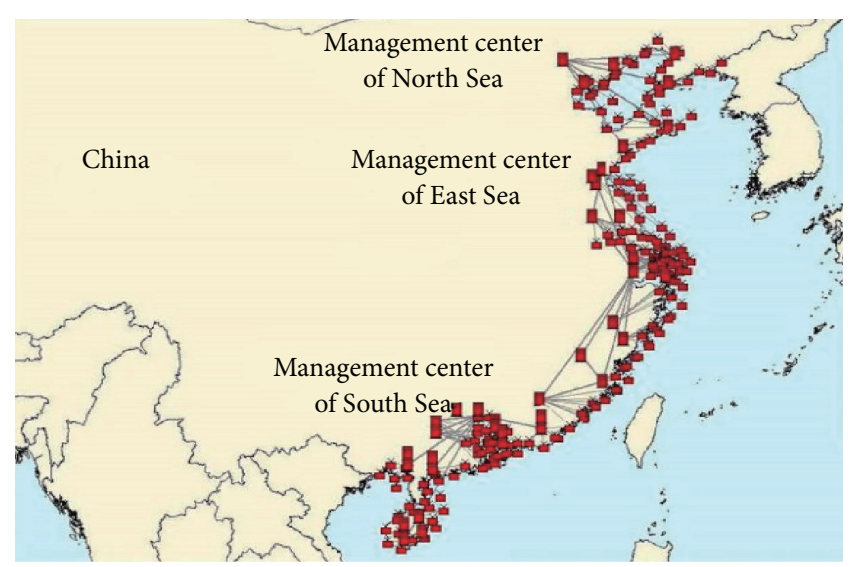

FIGURE 6: Coverage of national AIS shore-based stations.

\section{Positioning Error Analysis in China Sea Area}

5.1. Availability of Shore-Based Stations. Chinese shore-based AIS network system, which has been built by the MSA of China with investment of 500 million yuan since 2003, is one of the biggest fully operational, real-time systems with full routing capability in the world. The system consists of one AIS national management and maintenance center, three sea area management centers of the North China Sea, the East China Sea, and the South China Sea, thirty-three area management centers, and approximately 250 shore-based stations in hotstandby configurations, as shown in Figure 6. Currently, the signal coverage rate of Chinese coastline is higher than $99.97 \%$ and the reliability is higher than $99.95 \%$ [29]. It plays an important role in improving the safety and security of vessels and port facilities in China. Additionally, the further project of the Chinese shore-based AIS network system has still been carried out by MSA of China at present. There will be more AIS shore-based stations in China in the future.

The transmission distance of AIS signals can reach about $90 \mathrm{~km}$ under good sea condition. The signal coverage in the North China Sea, the South China Sea, and the East China Sea is shown in Figure 7. It can be seen that there are a number of the AIS shore-based stations in traffic dense areas. Therefore, there are areas where AIS signals can be received from three different shore-based stations. However, there are areas with insufficient shore-based stations as the reference nodes for positioning. Therefore, to further carry out the RMode positioning system using AIS signals for E-navigation in China, some of the probable solutions are presented in the following. Firstly, the most straightforward method is to consider establishing more AIS shore-based stations as positioning reference nodes. The second solution is to make use of AIS aids to navigation (AtoN). The AIS AtoN provides the position and status of buoys and lights through the same VDL with shipborne AIS device. Vessels around the AIS AtoN could receive signals transmitted by them. Thus, the AIS AtoN can be used as the reference nodes for R-Mode positioning based on AIS. In addition, the MF DGPS/DGNSS reference and monitoring stations or reference stations of 


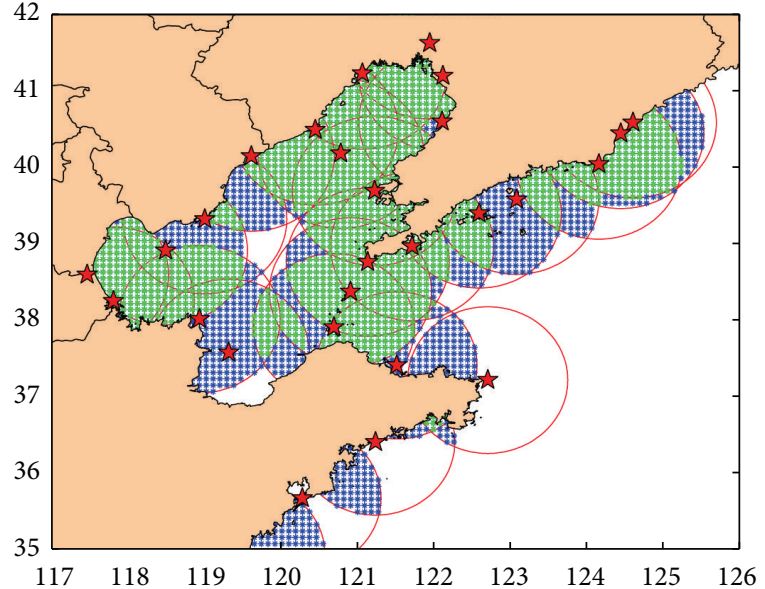

(a) North China Sea

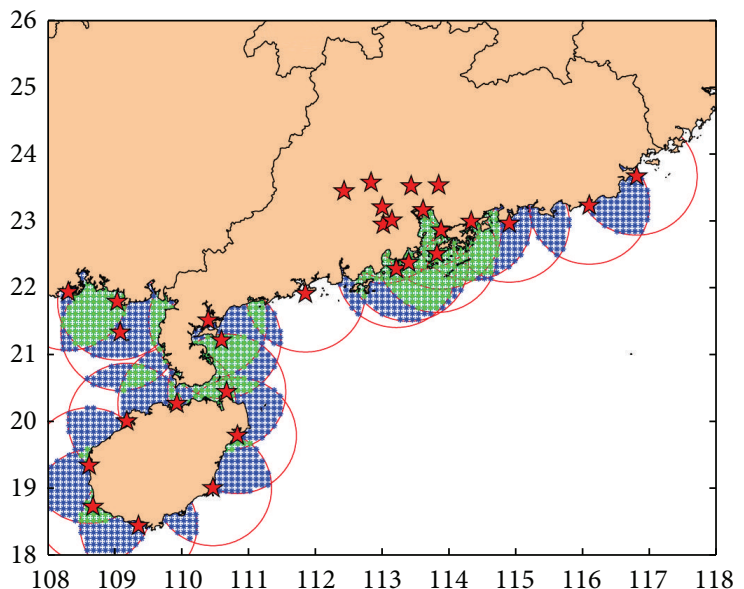

(b) South China Sea

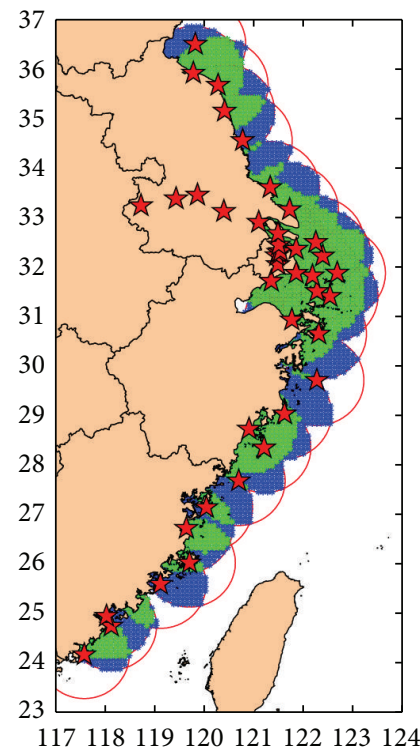

(c) East China Sea

FIGURE 7: Signal coverage of national AIS shore-based stations.

Changhe II system could also be considered for increasing the number of the positioning reference nodes.

5.2. Measurement Accuracy Evaluation. Figure 8 shows standard deviation of time estimation error of bit transition as a function of transmission distances and observation time. At the transmission distance of $90 \mathrm{~km}$, the standard deviation of measurement error is $8.5 \mathrm{~m}$ when the number of observation slots is 5; the standard deviation is $3.8 \mathrm{~m}$ when the number of slots is 1 . At the transmission distance of $45 \mathrm{~km}$, the standard deviation of measurement error is $4.2 \mathrm{~m}$ when the number of observation slots is 5 ; the standard deviation is $1.9 \mathrm{~m}$ when the number of slots is 1 . Therefore, the observation measured data in the shipborne AIS device should be as long as possible for improving the performance. However, as AIS signals use a TDMA scheme to share all time slots, the observation measured data could not be too long.
5.3. Positioning Accuracy Analysis. According to Section 4, the performance of TDOA is better than TOA in terms of DOP. Therefore, the following discussion in this paper is focused on the TDOA technology. Figure 9 shows the positioning error distribution of AAPS in the North, East, and South Sea regions of China. The general transmission distance of $90 \mathrm{~km}$ is taken as an example in Figure 9. The measurement error and DOP are both considered to evaluate the positioning accuracy. As the availability of shore-based stations, which have been investigated before, position information could not be provided by AAPS in some area. However, it can be seen that the positioning accuracy is good enough to meet the requirements for vessel navigation in the regions that AIS signals from three shorebased stations cover. Particularly worth mentioning is the positioning accuracy at the meter level in most regions. With the promotion of R-Mode positioning system, there would be 


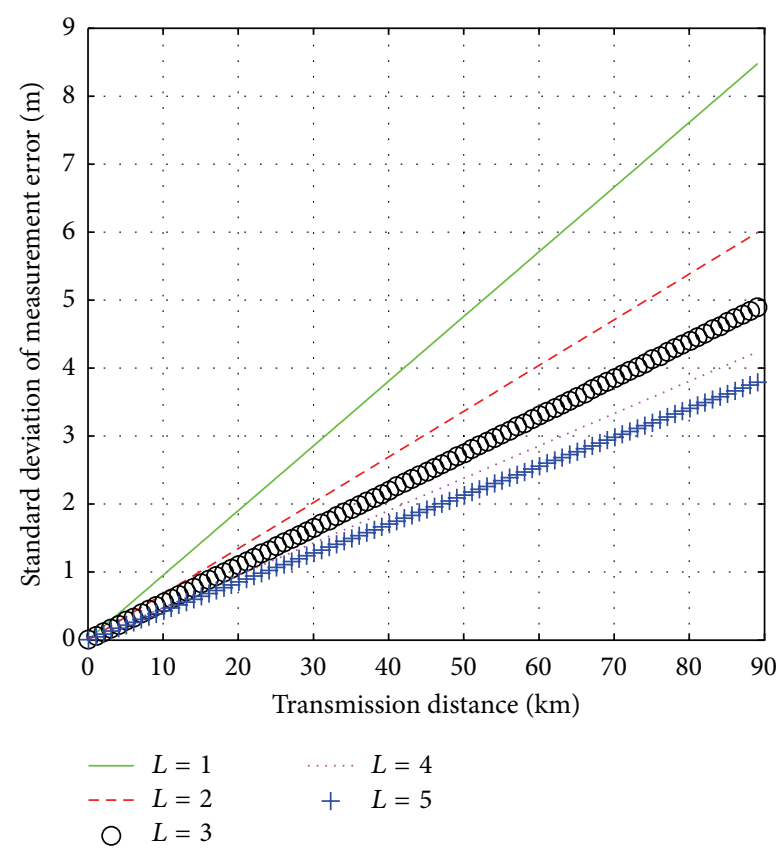

Figure 8: Performance of measurement accuracy.

TABLE 1: Location of shore-based stations.

\begin{tabular}{lcc}
\hline Station name & Latitude & Longitude \\
\hline Lingjing & $38^{\circ} 50^{\prime} 21.309^{\prime \prime} \mathrm{N}$ & $121^{\circ} 30^{\prime} 46.005^{\prime \prime} \mathrm{E}$ \\
Heishijiao & $38^{\circ} 51^{\prime} 58.689^{\prime \prime} \mathrm{N}$ & $121^{\circ} 33^{\prime} 05.779^{\prime \prime} \mathrm{E}$ \\
Fujiazhuang & $38^{\circ} 51^{\prime} 52.660^{\prime \prime} \mathrm{N}$ & $121^{\circ} 36^{\prime} 49.543^{\prime \prime} \mathrm{E}$ \\
\hline
\end{tabular}

more positioning reference nodes. The DOP will be smaller, positioning coverage will be larger, and the positioning accuracy will be better.

5.4. Experimental Results Analysis. A positioning testbed of AAPS has been set up in Xinghai Bay of Dalian, China, to verify the real-time positioning under real condition [11]. Three shore-based stations have been established as the reference nodes. Considering that the positioning testbed should be convenient for experiment, the shore-based stations were established closely. Table 1 gives location about these reference nodes.

Figure 10 evaluates the distribution of these reference nodes in the testbed of AAPS according to the DOP. The reference nodes are denoted by the red stars. Heishijiao station is a master node; Lingjing station and Fujiazhuang station are the slave nodes. The baselines are relatively short, which are $4.511 \mathrm{~km}$ and $5.38 \mathrm{~km}$. In the range of about $9 \mathrm{~km}$ from Heishijiao station, DOP is less than 5. However, the DOP distribution in the real China sea area is much better, as the baseline of the Chinese shore-based AIS network system is longer.

In Figure 10, two locations in the above positioning range denoted by the purple diamonds are selected to carry out the positioning experiments, whose latitude and longitude are shown in Table 2. The below purple diamond in Figure 10
TABLE 2: Location information of experiment.

\begin{tabular}{lcc}
\hline Name & Latitude & Longitude \\
\hline Laopian Island & $38^{\circ} 47^{\prime} 43.402^{\prime \prime} \mathrm{N}$ & $121^{\circ} 35^{\prime} 50.102^{\prime \prime} \mathrm{E}$ \\
Mooring & $38^{\circ} 50^{\prime} 24.837^{\prime \prime} \mathrm{N}$ & $121^{\circ} 33^{\prime} 35.142^{\prime \prime} \mathrm{E}$ \\
\hline
\end{tabular}

TABLE 3: Experiment results.

\begin{tabular}{lcc}
\hline Name & Laopian Island & Mooring \\
\hline Latitude error $(\mathrm{m})$ & 16.552 & 4.319 \\
Longitude error $(\mathrm{m})$ & 9.502 & 2.228 \\
Position error $(\mathrm{m})$ & 19.085 & 4.859 \\
DOP & 4.551 & 1.191 \\
\hline
\end{tabular}

indicates Laopian Island; the above one indicates the location where ship was moored. The boat we used in the positioning experiments is shown in Figure 11(a). The shipborne AIS position device which we have developed and implemented is shown in Figure 11(b).

The scenario of the position experiments is shown in Figure 12. Figure 12(a) is Laopian Island. Figure 12(b) is the environment of the position experiment when ship was moored.

Figures 13(a) and 13(b) show the positioning results of the Laopian Island and mooring, respectively. The positioning errors are given in Table 3 in detail. Here, the error means the root mean square (RMS) of the difference between the position results output from the shipborne AIS positioning device and the position data from the continuously operating reference station (CORS) system, the position accuracy of which is centimeter level. We can see that the measurement error is larger than the CRB derived theoretically in Section 3, because the ASFs, the multipath, and other interference cannot be ignored in the real condition. However, under the same conditions of the measurement, the position error of the Laopian Island and mooring is corresponding to their DOP. In conclusion, the experimental results are consistent with the theoretical analysis.

\section{Conclusions}

The R-Mode positioning using AIS signals is the effective solution for the resilient PNT of E-Navigation. The Chinese shore-based AIS network system is one of the biggest in the world. It is more probable and feasible to carry out AAPS in China. As the accuracy is the key for the positioning system, the position error of the R-Mode positioning based on AIS shore-based stations in China is investigated in this paper. Firstly, the principle of R-Mode positioning using AIS signals is given. Then measurement errors of GMSK demodulation based on carrier phase locking loop are analyzed in theory. Moreover, DOP for TOA and TDOA used in AAPS are discussed in two conditions with different measurement mechanisms. Finally, the positioning error distributions in the North, East, and South Sea regions of China based on the existing AIS shore-based stations are evaluated. The theoretical analysis is verified by the real positioning experiments 


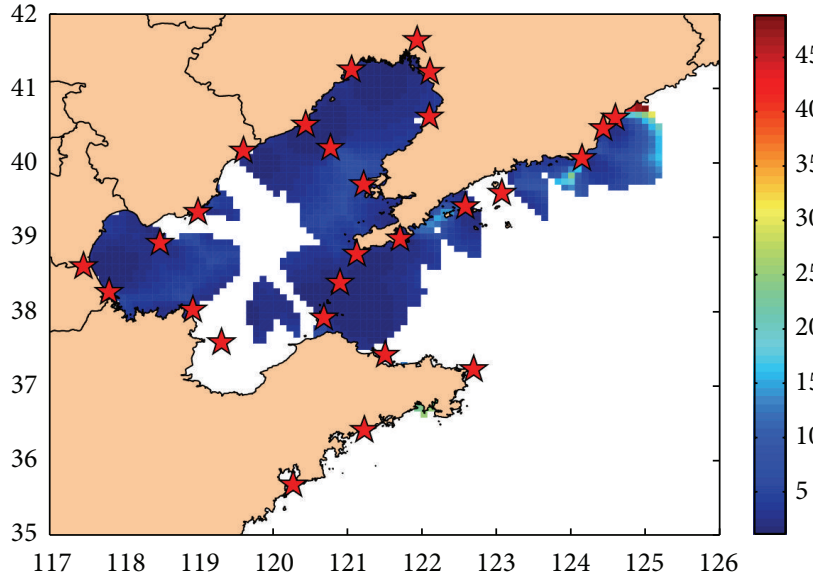

(a) North China Sea

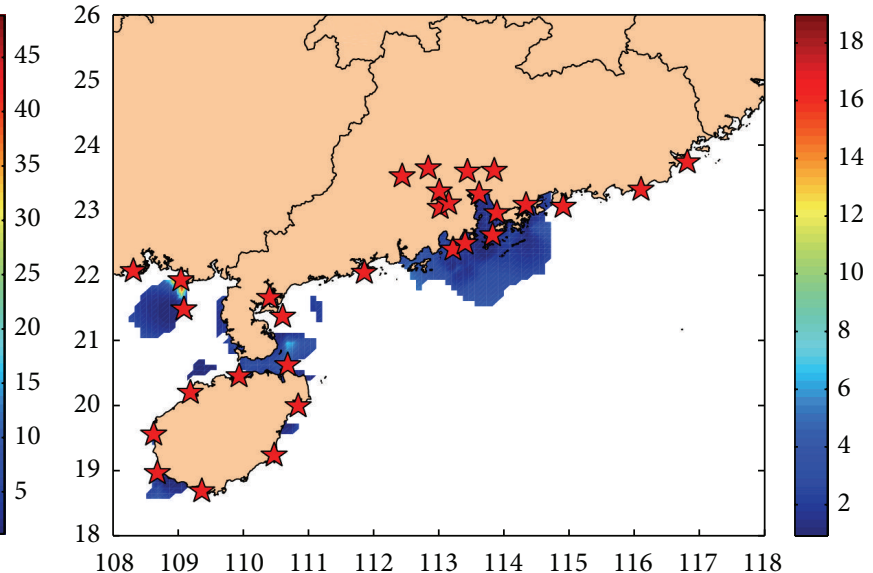

(b) South China Sea

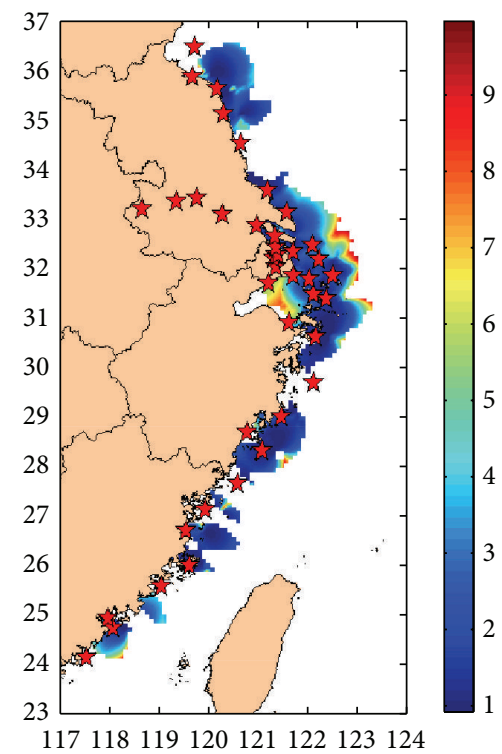

(c) East China Sea

Figure 9: Positioning accuracy in three main sea regions.

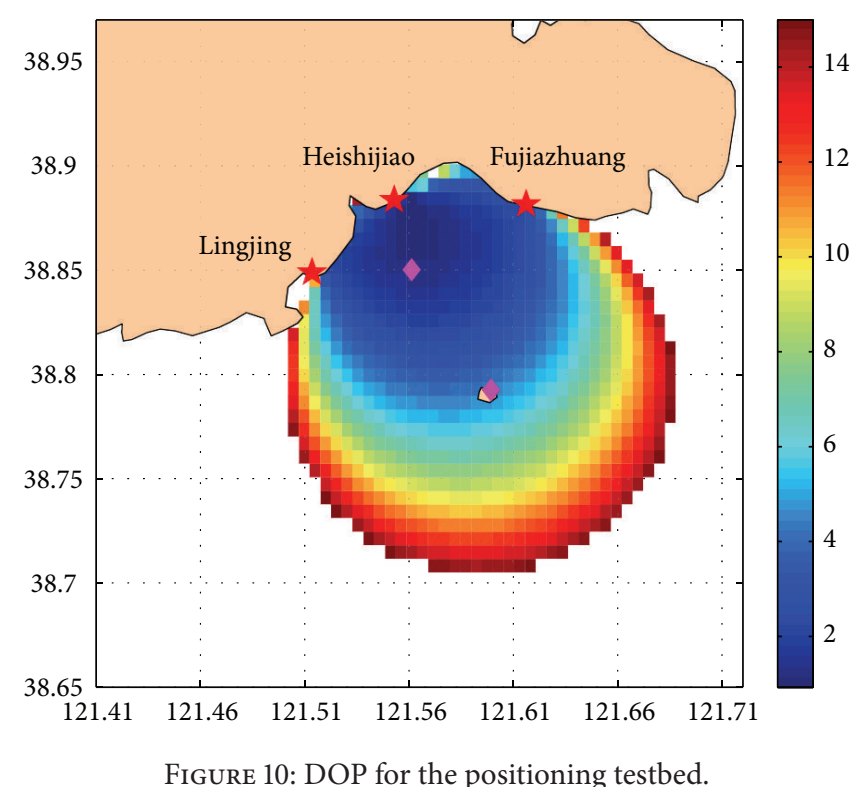

FIGURE 10: DOP for the positioning testbed. 


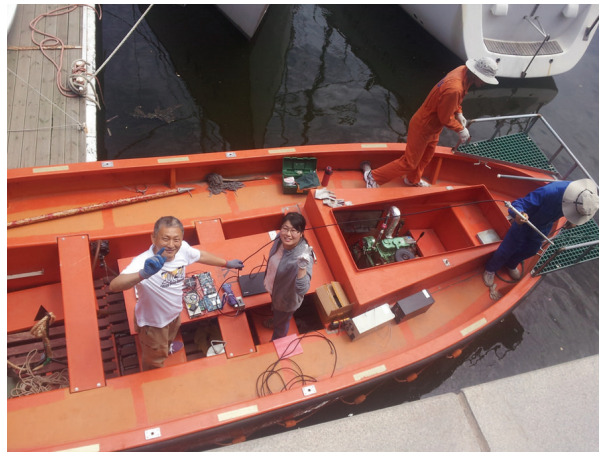

(a)

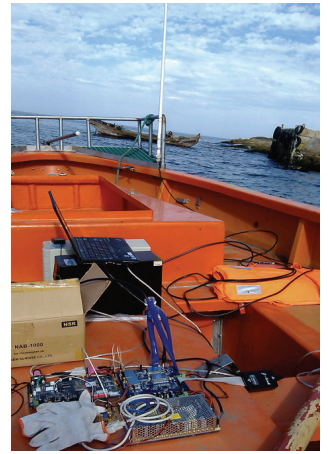

(b)

Figure 11: Device of positioning experiment.

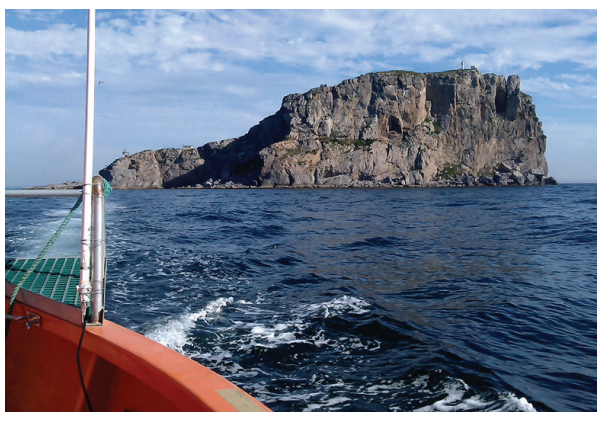

(a) Laopian Island

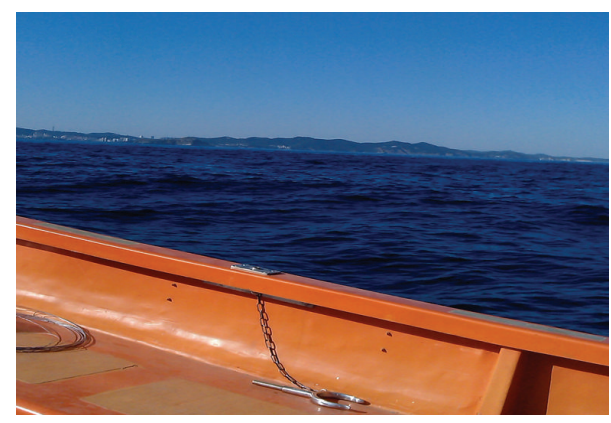

(b) Mooring

FIGURE 12: Positioning experiment scenario.

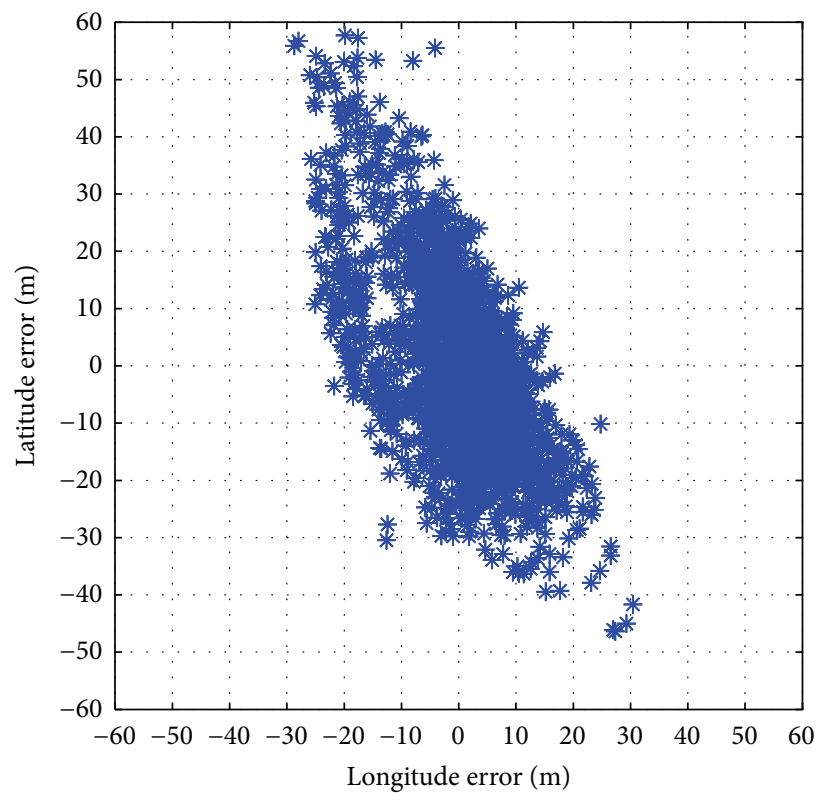

(a) Laopian Island

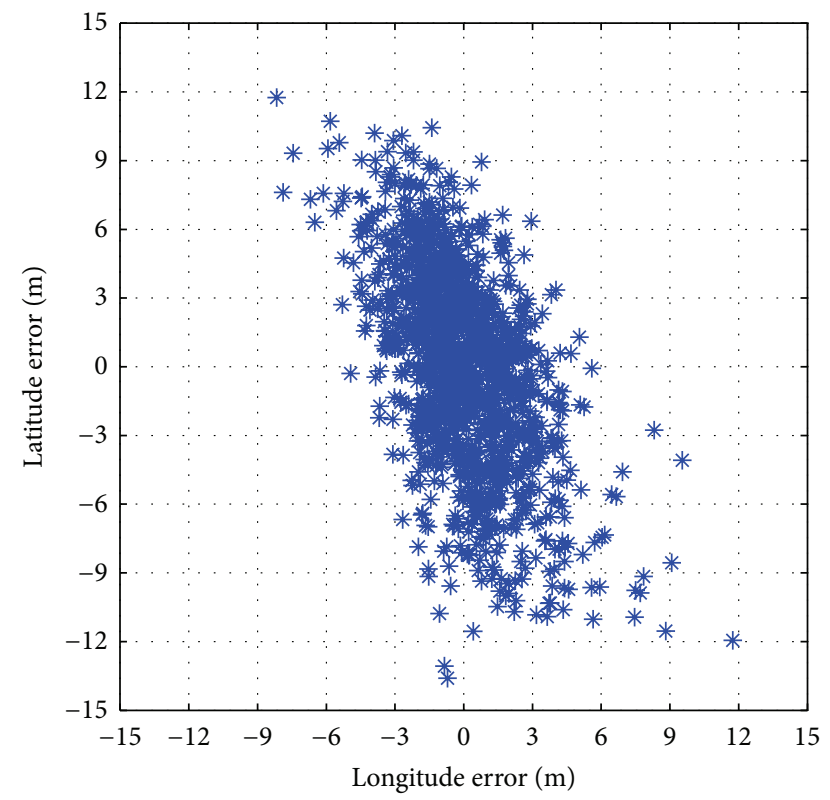

(b) Mooring

FIGURE 13: Positioning errors for the static position. 
of AAPS testbed further. The positioning accuracy is at the meter level in the most traffic dense areas to meet the requirements for vessel navigation.

\section{Competing Interests}

The authors declare that the funding mentioned in the Acknowledgments does not lead to any conflict of interests. Additionally, the authors declare that there is no conflict of interests regarding the publication of this manuscript.

\section{Acknowledgments}

This work was supported by the Chinese National Science Foundation (nos. 61231006 and 61501079), Foundation of Liaoning Educational Committee (nos. L2013197 and L2015059), Liaoning Provincial Natural Science Foundation (nos. 2014025002 and 2014025009), and the Fundamental Research Funds for the Central Universities (no. 3132016317).

\section{References}

[1] P. Williams, A. Grant, C. Hargreaves, M. Bransby, N. Ward, and D. Last, "Resilient PNT for e-navigation," in Proceedings of the ION 2013 Pacific PNT Meeting, pp. 477-484, Honolulu, Hawaii, USA, April 2013.

[2] A. Grant, P. Williams, G. Shaw, M. De Voy, and N. Ward, "Understanding GNSS availability and how it impacts maritime safety," in Proceedings of the Institute of Navigation International Technical Meeting, pp. 687-695, San Diego, Calif, USA, January 2011.

[3] N. Ward, G. Shaw, P. Williams, and A. Grant, "The Role of GNSS in E-navigation and the Need for Resilience," 2010, http://www .forschungsinformationssystem.de/servlet/is/342313/.

[4] P. Williams, A. Grant, and C. Hargreaves, "Resilient PNTmaking way through rough waters," in Proceedings of the 7th GNSS Vulnerabilities and Solutions Conference, September 2013.

[5] International Association of Lighthouse Authorities, IALA Worldwide Radio Navigation Plan, Version 2, IALA, 2012.

[6] International Maritime Organization, SOLAS 1974 Amendments, The Bath Press, Bath, UK, 2000.

[7] https://www.amsa.gov.au/navigation/services/ais/.

[8] http://www.navcen.uscg.gov/?pageName=NAISmain.

[9] L. J. Foged, A. Giacomini, F. Saccardi et al., "Miniaturized array antenna using artificial magnetic materials for satellite-based AIS system," IEEE Transactions on Antennas and Propagation, vol. 63, no. 4, pp. 1276-1287, 2015.

[10] X. D. Zhang, "World's largest AIS Shore-based network in China," Marine Technology, vol. 5, p. 55, 2011.

[11] G. Johnson and P. Swaszek, Feasibility Study of R-Mode Using AIS Transmissions, 2014, http://www.accseas.eu/publications/ r-mode-feasibility-study/.

[12] G. Johnson, P. Swaszek, J. Alberding, M. Hoppe, and J.-H. Oltmann, "The feasibility of R-mode to meet resilient PNT requirements for e-navigation," in Proceedings of the 27th International Technical Meeting of the Satellite Division of the Institute of Navigation (ION GNSS '14), pp. 3076-3100, Tampa, Fla, USA, September 2014.
[13] S. F. Zhang and Q. Hu, "Ship Autonomous Positioning System based on Automatic Identification System (AIS)," CN Patent 102305936B, July 2013.

[14] Y. Jiang, S. F. Zhang, and D. K. Yang, "A novel position estimation method based on displacement correction in AIS," Sensors, vol. 14, no. 9, pp. 17376-17389, 2014.

[15] Q. Hu, W. Hu, S. F. Zhang, and J. B. Zhang, "An improved candidate slot selection method for AIS," CN Patent 103178921A, 2016.

[16] Q. Hu, Y. Jiang, J. B. Zhang, X. W. Sun, and S. F. Zhang, "Development of an automatic identification system autonomous positioning system," Sensors, vol. 15, no. 11, pp. 28574-28591, 2015.

[17] J. Zhen, X.-H. Ma, and S.-F. Zhang, “TOA-based combined location positioning algorithm in NLOS propagation environment," Journal of Dalian University of Technology, vol. 45, no. 4, pp. 599-602, 2005.

[18] H. K. Lee, H.-S. Kim, J.-Y. Shim, and M. B. Heo, "Analytic equivalence of iterated TOA and TDOA techniques under structured measurement characteristics," Multidimensional Systems \& Signal Processing, vol. 22, no. 4, pp. 361-377, 2011.

[19] Y. Jiang, Q. Hu, and D. K. Yang, "Analysis of positioning error for two-dimensional location system," Mathematical Problems in Engineering, vol. 2013, Article ID 163958, 8 pages, 2013.

[20] Y. Jiang, S. F. Zhang, and D. K. Yang, "A general method for evaluation of position accuracy," ICIC Express Letters, vol. 7, no. 7, pp. 2035-2040, 2013.

[21] P. Williams and C. Hargreaves, "UK eLoran-initial operational capability at the port of Dover," in Proceedings of the International Technical Meeting of The Institute of Navigation, pp. 392402, January 2013.

[22] C. Kim, T. Lee, H. So et al., "A new approach to calibrate pseudolite position using multi-antenna and carrier phase measurement without cycle ambiguity," in Proceedings of the International Technical Meeting of the Institute of Navigation, pp. 250-257, San Diego, Calif, USA, January 2011.

[23] International Telecommunication Union, Technical Characteristics for a VHF Data Exchange System in the VHF Maritime Mobile Band, ITU, 2015.

[24] International Telecommunication Union, Technical Characteristics for an Automatic Identification System Using Time Division Multiple Access in the VHF Maritime Mobile Frequency Band, ITU, 2014.

[25] J. B. Zhang, W. Hu, and Q. Hu, "Novel Carrier Extraction Method for Binary Frequency Shift Keying Signals," CN Patent 103457892A, December 2013.

[26] U. Mengali and A. N. D’Andrea, Synchronization Techniques for Digital Receivers, Springer, Boston, Mass, USA, 1997.

[27] International Telecommunication Union, Calculation of FreeSpace Attenuation, 1994.

[28] International Telecommunication Union, Interim Solutions for Improved Efficiency in the Use of the Band 156-174 MHz by Stations in the Maritime Mobile Service, 2012.

[29] http://www.moc.gov.cn/2011wangshangzhibo/shibiexitong/ 201512/t20151226_1965809.html. 


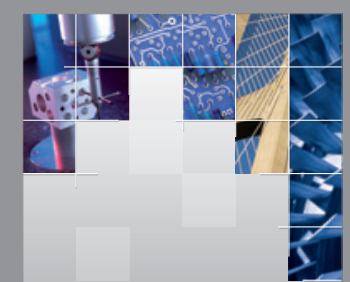

\section{Enfincering}
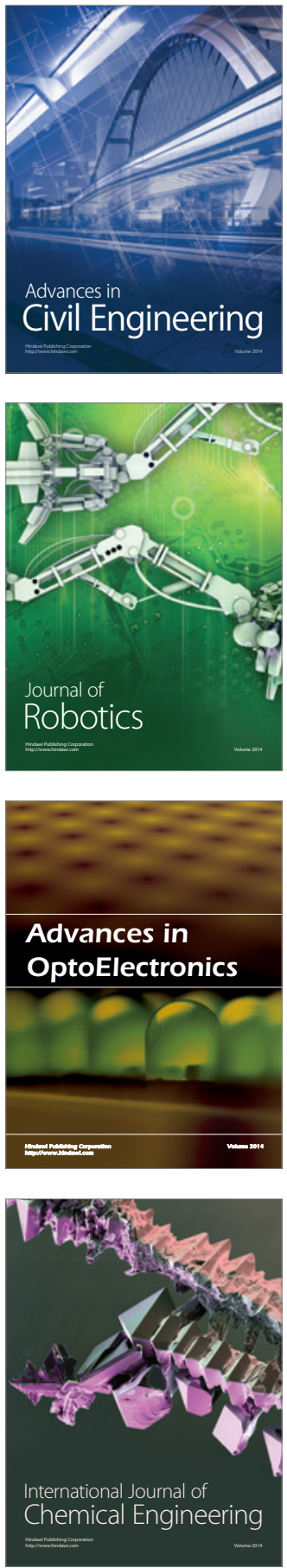

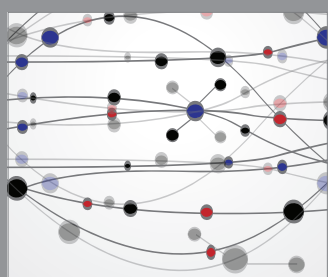

The Scientific World Journal

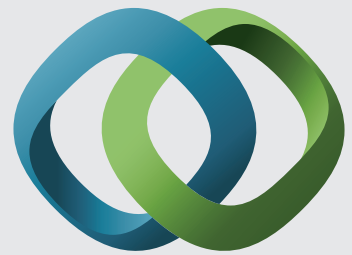

\section{Hindawi}

Submit your manuscripts at

http://www.hindawi.com
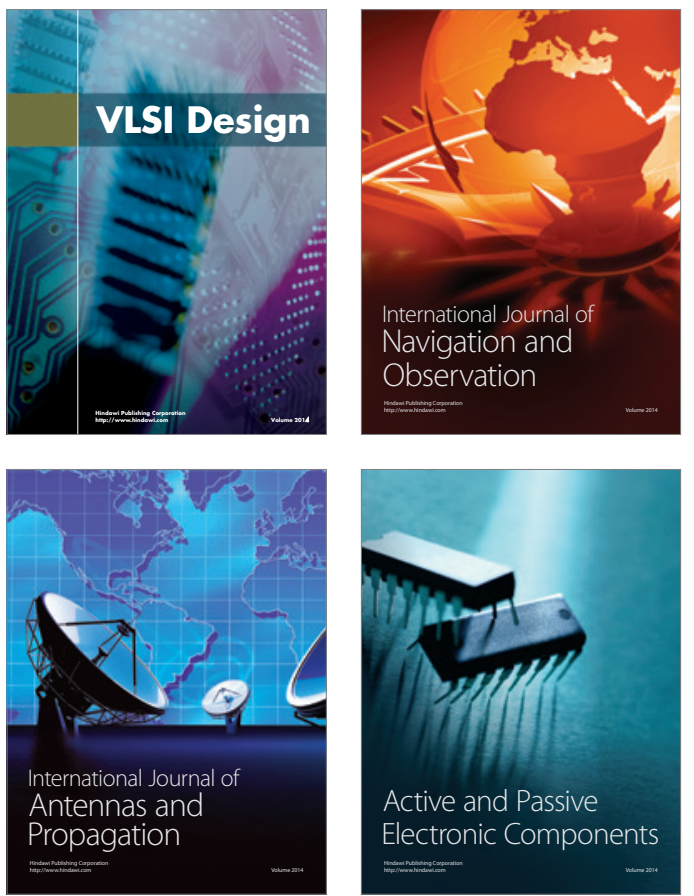
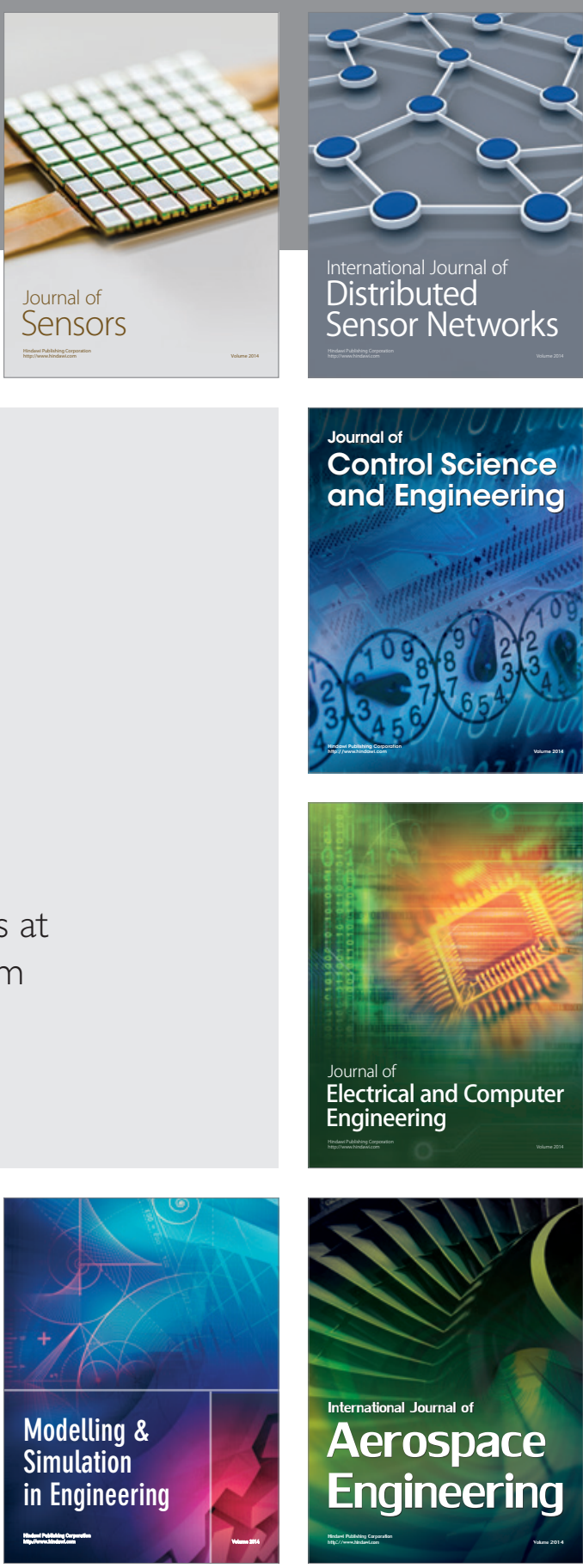

International Journal of

Distributed

Sensor Networks

Journal of

Control Science

and Engineering
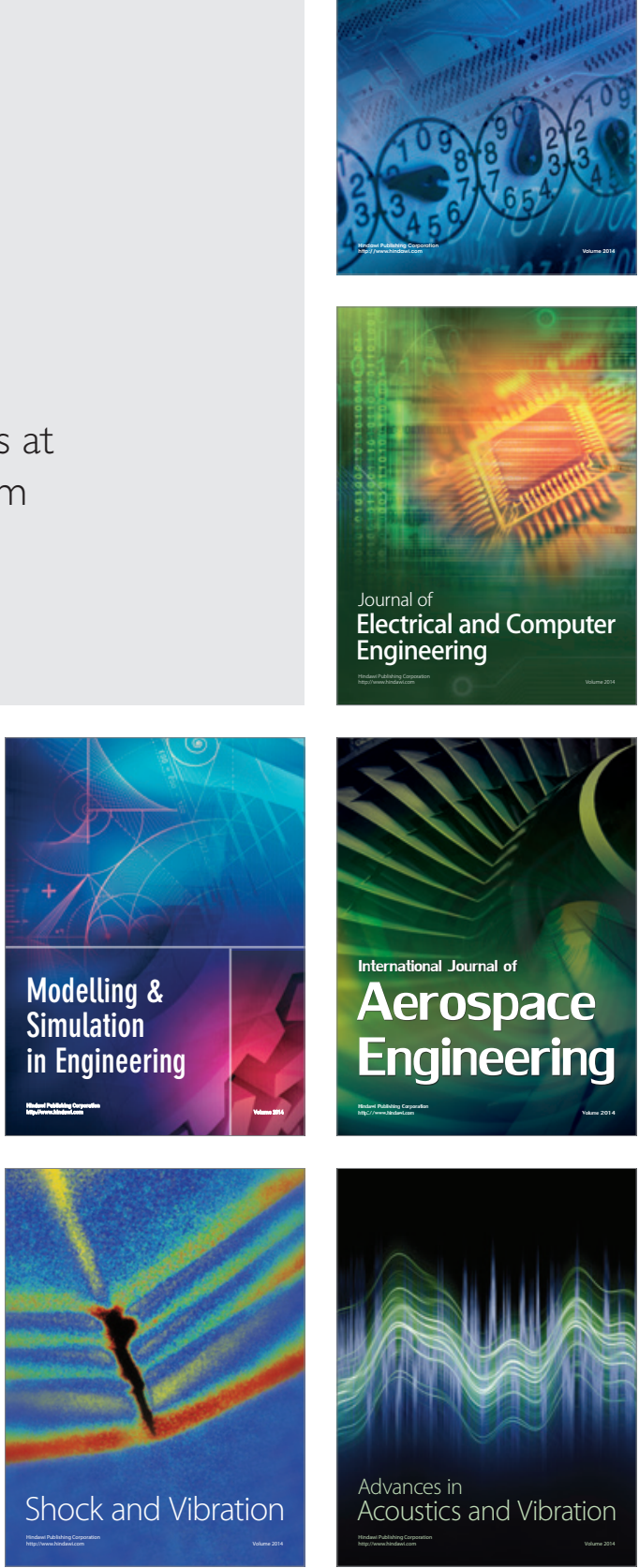